\title{
Misdirected Quasars in distant Radio Galaxies
}

\author{
Sperello di Serego Alighieri \\ Osservatorio Astrofisico di Arcetri \\ Largo E. Fermi 5, I-50125, Firenze, Italy \\ and \\ Andrea Cimatti \\ European Southern Observatory \\ Karl-Schwarzschild Str.2, D-8046, Garching bei München, Germany
}

\begin{abstract}
We present the results of recent spectro-polarimetry and imaging-polarimetry of distant radio galaxies which show: (1) broad polarized permitted emission lines, (2) narrow unpolarized forbidden emission lines, (3) a flat (in $f_{\lambda}$ ) polarized UV continuum and (4) an absorption feature, probably interstellar. The direction of the $E$ vector of polarization is always perpendicular to the optical/radio axis. These observations are strong evidence that these objects harbour a quasar, which is visible only through scattering by the interstellar medium of the galaxy. The continuum polarization drops to the red of the $4000 \AA$ break, suggesting dilution by an evolved stellar population. A two-component model made of a dust scattered quasar and an evolved stellar population reproduces well the polarization and the spectral energy distribution, including the IR data.
\end{abstract}

\section{Introduction}

The unified models of Active Galactic Nuclei (AGN, see [1] for a recent review) foresee that a type 1 spectrum, consisting of a featureless continuum and broad emission lines, is emitted anisotropically from the nuclear regions in two opposite cones and is visible directly only if our line of sight falls in the cones. For misdirected objects an indirect view of the central regions is provided by scattering, as has been beautifully demonstrated by the presence of a polarized type 1 spectrum for a few nearby AGN (e.g. NGC 1068, [11]). For the more distant radio loud AGN the unified model is based on the evidence for relativistic beaming in quasars which may well not be the only source of anisotropy - and on a statistical analysis of the angular size of a complete sample of extragalactic powerful radio sources [2], leaving it as an open question whether the unbeamed parent population is made of radio galaxies.

The discovery of perpendicular polarization in distant radio galaxies ([7] and [4] for a recent review) is a strong indication of the presence of scattered nuclear radiation, but, since it results from broad-band imaging polarimetry, it gives little information on the scattered light, not allowing, for example, to decide whether it has a quasar- or a blazar-like spectrum. We have therefore started a programme of spectro-polarimetry of distant radio galaxies, with the aim of studying the nature of the scattered radiation, understanding the scattering mechanism and disentangling the spectral energy distribution of the stellar radiation. We present here the first results for two radio galaxies with redshift around one.

21

T. J.-L. Courvoisier and A. Blecha: Multi-Wavelength Continuum Emission of AGN, 21-24.

(C) 1994 IAU. Printed in the Netherlands. 


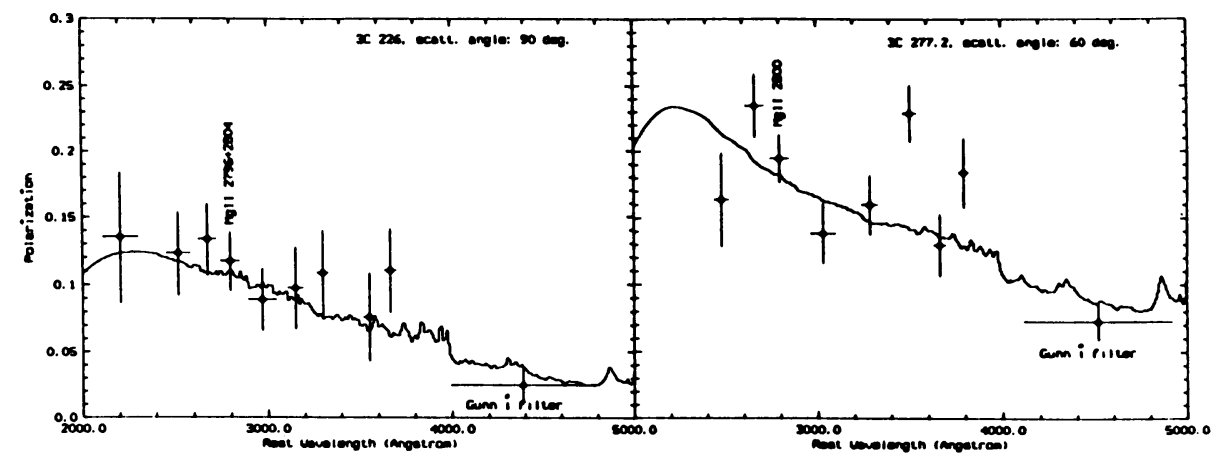

Fig. 1. The degree of polarization of the continuum radiation. The data for a band across the broad MgII $\lambda 2800$ line are also shown. The horizontal "error" bars represent the band over which the measurements have been performed. The continuous line shows the polarization foreseen by our two-component model.

\section{Observations and Results}

The results of recent imaging- and spectro-polarimetry of $3 \mathrm{C} 226(\mathrm{z}=0.818)$ and 3C $277.2(\mathrm{z}=0.766)$, obtained with the ESO Faint Object Spectrograph and Camera, are the following:

The MgII $\lambda 2800$ line is clearly broader than the forbidden lines, like $[\mathrm{NeV}] \lambda 3426$, with a relative broadening of $3700 \mathrm{~km} / \mathrm{s}$ (FWHM). The width of the line corresponds well to that measured for the average spectrum of radio loud quasars [5]. The continuum is almost flat in $f_{\lambda}$. A clear absorption feature is present at $2600 \AA$ in all individual spectra.

The polarization of the continuum was measured on the spectra for wavelength bins carefully selected to avoid emission lines and strong sky features, and is shown in Fig. 1 together with the polarization obtained in the Gunn $i$ band with imaging polarimetry. The polarization is high (10-20\%) and almost flat between 2000 and $4000 \AA$, and suddenly drops beyond $4000 \AA$ to a much lower but still significant value. The polarization over a band across the broad $\operatorname{MgII} \lambda 2800$ line is also shown in Fig. 1: it is high, equal to that of the surrounding continuum. Since the equivalent width of the MgII $\lambda 2800$ line is large $(\sim 50 \AA)$, the line itself must be polarized at a level close to that of the continuum. The direction of the $E$ vector is constant with wavelength and perpendicular to the optical/radio axis. The narrow forbidden lines are not polarized for both galaxies. 


\section{Discussion}

The results presented here are strong evidence that the two distant radio galaxies observed harbour a quasar, which is not visible directly, but shines its Type 1 spectrum into two opposite cones along the radio axis, where this radiation is scattered in our direction by the interstellar medium of the host galaxy, thereby confirming the beaming/scattering idea [12] to explain the alignment effect in distant radio galaxies, and already supported by the results of imaging polarimetry ([4] and references therein). The main evidence comes from the presence of a polarized type 1 spectrum, which has a continuum approximately flat in $f_{\lambda}$ and a broad MgII $\lambda 2800$ line. In addition the direction of the $E$ vector is perpendicular to the radio/optical axis at all wavelengths observed. Our results provide strong support for the radio loud quasars/radio galaxies unification.

The scattering medium could be dust [6] or electrons [8] or a mixture of both. Fabian [8] has foreseen that scattering by electrons in a hot halo would broaden the incident spectrum by about $30000 \mathrm{~km} \mathrm{~s}^{-1}$, effectively smearing out any spectral feature. The fact that broad polarized lines are observed at about the expected width for a quasar spectrum therefore rules out the possibility that scattering is due to hot electrons. Also the observed polarization is too low for electron scattering, while it is consistent with dust scattering.

The sudden drop in the degree of polarization observed both in $3 \mathrm{C} 226$ and $3 \mathrm{C} 277.2$ beyond $4000 \AA$ points to the presence of diluting radiation with a strong $4000 \AA$ break. We interpret this drop as due to dilution by the light of the host galaxy which contains an evolved stellar population. The possible presence of young stars is severely limited by the fact that the dilution at $2800 \AA$ must be small since the MgII line has a polarization close to that of the continuum.

The polarization of the MgII line indicates that, at least in this case, anisotropy must be procuced by obscuration, the broad line region being inside the obscuring material. On the other hand, the less dens gas producing the unpolarized narrow lines must emit isotropically and therefore be outside the obscuring material.

We interpret the absorption feature at $2600 \AA$ as due to interstellar FeII $\lambda 2600.18$, which is the strongest UV interstellar absorption line with $\operatorname{MgII} \lambda 2796+2804$ [9].

We have modelled the spectral energy distribution of the two galaxies, including the infrared photometric data [10], with a two component model made of a dust scattered quasar and an evolved stellar population. We have used the typical spectrum of a radio loud quasar [5] scattered by dust with a galactic composition and grain size distribution, adopting Mie single scattering [4]. For the stellar component we use the synthetic spectra of simple stellar populations (instantaneous burst) [3] and Salpeter IMF over a range of 0.1-125 $\mathrm{M}_{\odot}$. We have also introduced a certain amount of dust extinction, to decrease the UV light. Nevertheless at least part of this extinction is probably due to the fact that the scattering cross section that we have used has a strong $2200 \AA$ feature, while recent data show that this feature is only present in absorption, but not in scattering. 


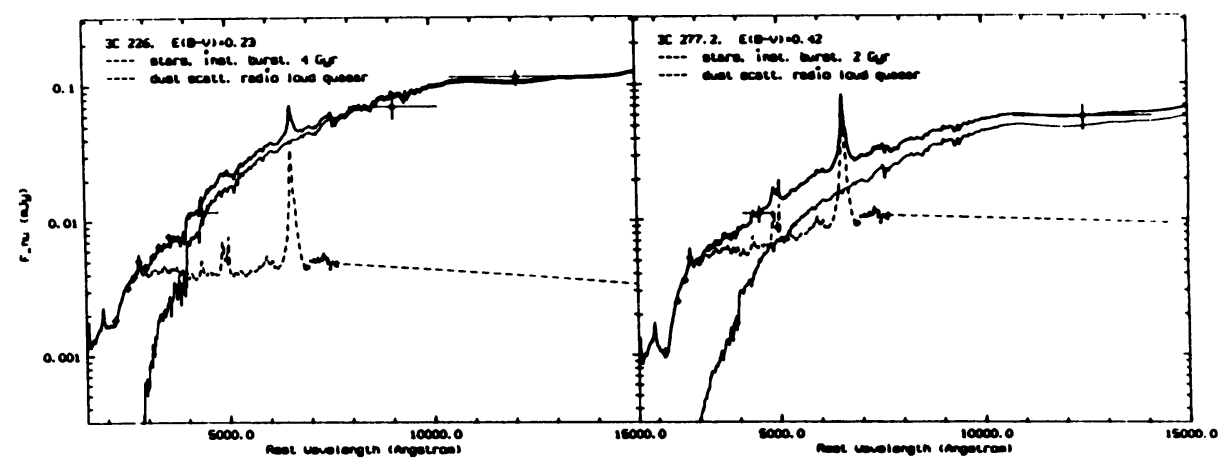

Fig. 2. The SED of $3 \mathrm{C} 226$ and $3 \mathrm{C} 277.2$, compared with our two-component model.

The results of our spectral models are shown in Fig. 2, which give also the amount of extinction and the age of the stellar population. Clearly the model fits the data of both galaxies very well $\left(\chi^{2}{ }_{r e d}=1.17\right.$ and 1.31 for $3 \mathrm{C} 226$ and $3 \mathrm{C} 277.2$ respectively). The age of the stellar population that give an acceptable fit is limited to be larger than $3 \mathrm{Gyr}$ for $3 \mathrm{C} 226$ and to be between 0.5 and 3 Gyr for 3C 277.2. The presence of an evolved stellar population is also consistent with the drop of the polarization beyond $4000 \AA$. The polarization predicted by our model for the total light is shown in Fig. 1 and is consistent with the observed values. With the advent of 8-10 m class telescopes similar work can be extended to a larger sample and will provide a more precise definition of the incident quasar spectrum, of the scattering parameters, and of the age of the stellar population.

\section{References}

1. Antonucci R., 1993, $A R A A, 31,473$

2. Barthel P.D., 1989, ApJ, 336, 606

3. Bruzual G., Charlot S. 1993, $A p J, 405,538$

4. Cimatti A., di Serego A. S., Fosbury R.A.E., Salvati M., Taylor D. 1993, MNRAS, in press

5. Cristiani S., Vio R., 1990, $A \& A, 227,385$

6. di Serego Alighieri S., Binette L., Courvoisier T., Fosbury R.A.E., Tadhunter C.N., 1988, Nature, 334, 591

7. di Serego Alighieri S., Fosbury R.A.E., Quinn P.J., Tadhunter C.N., 1989, Nature, 341, 307

8. Fabian A.C., 1989, MNRAS, 238, 41p

9. Kinney A.L., Bohlin R.C., Calzetti D., Panagia N., Wise R.F.G., 1993, ApJ Suppl., in press

10. Lilly S.J., Longair M.S., 1984, MNRAS, 211, 833

11. Miller J.S., Goodrich R.W., Mathews W., 1991, ApJ, 378, 47

12. Tadhunter C.N., Fosbury R.A.E., di Serego Alighieri S., 1989, in Maraschi L., Maccacaro T. \& Ulrich M.H., eds., BL Lac Objects, Springer-Verlag, Berlin, p. 79 